Geração de resíduos sólidos e elaboração de um sistema de gerenciamento usando análise estatística

Generation of solid waste and development of a management system using statistical analysis Generación de residuos sólidos y desarrollo de un sistema de gestión mediante análisis estadístico

Recebido: 10/02/2020 | Revisado: 13/02/2020 | Aceito: 29/02/2020 | Publicado: 12/03/2020

\title{
Caroline Coutinho
}

ORCID: https://orcid.org/0000-0002-2796-7161

Universidade do Estado do Rio de Janeiro, Brasil E-mail: caroline.uerj.fat@gmail.com

Allan Duarte ORCID: https://orcid.org/0000-0001-5494-7847 Universidade do Estado do Rio de Janeiro, Brasil E-mail: allannduarte@gmail.com Emanuel Carneiro ORCID: https://orcid.org/0000-0001-5818-3346 Universidade do Estado do Rio de Janeiro, Brasil E-mail: emanuel.carneiro1998@gmail.com Nilo Antonio Souza Sampaio ORCID: https://orcid.org/0000-0002-6168-785X Universidade do Estado do Rio de Janeiro, Brasil E-mail: nilo.samp@ @erra.com.br José Wilson de Jesus Silva ORCID: https://orcid.org/0000-0002-0033-2270 Centro Universitário Teresa D’Ávila, Brasil E-mail: jwjsilva@gmail.com Carin Von Mühlen ORCID: https://orcid.org/0000-0003-0876-1237 Universidade do Estado do Rio de Janeiro, Brasil E-mail: carinvm@gmail.com 
Com o objetivo de desenvolver novas tecnologias que impactem diretamente na realidade ambiental do Brasil no âmbito do gerenciamento de resíduos sólidos e na educação socioambiental, o projeto Síntese é desenvolvido pelo Departamento de Engenharia Química e Ambiental e por alunos do curso de Engenharia Química da Faculdade de Tecnologia da Universidade do Estado do Rio de Janeiro, Brasil. Para tal, utiliza-se como grupo focal a população do próprio campus regional de Resende, onde o projeto é desenvolvido. Com base nos tipos de resíduos gerados pelo campus, são propostas alternativas para o tratamento dos mesmos, desenvolvimento de tecnologias que possam ser aplicadas à realidade da sociedade brasileira e de medidas que estimulem a sociedade a fazer parte do processo de tratamento dos resíduos, gerando um impacto social e de responsabilidade ambiental. A etapa inicial do trabalho desenvolvido pelo projeto é o levantamento dos tipos de resíduos gerados pela população do campus para que sejam determinadas as áreas de atuação prioritárias para o desenvolvimento de soluções e vertentes tecnológicas. Após esta etapa, são determinadas divisões de desenvolvimento para cada grande grupo de resíduo do campus, posteriormente visando realidade industrial da região. Foi observado que a maior parte dos resíduos gerados eram de materiais recicláveis, seguido de materiais não recicláveis, assim como o observado em outras universidades, a maior divergência se deu na discrepância de material compostável recolhido.

Palavras-chave: Ambiental; Tecnologia; Universidade; Compostável.

\begin{abstract}
Aiming to develop new technologies that directly impact Brazil's environmental reality in the scope of solid waste management and socio-environmental education, the Synthesis project is developed by the Department of Chemical and Environmental Engineering and by students from the Chemical Engineering course at the Faculty of Technology at the State University of Rio de Janeiro, Brazil. To this end, population of Resende regional campus, where the project is developed, is used as a focus group. Based on the types of waste generated by the campus, alternatives are proposed for their treatment, development of technologies that can be applied to the reality of Brazilian society and measures that encourage society to be part of the waste treatment process, generating a social impact and environmental responsibility. The initial stage of the work developed by the project is the survey of types of waste generated by the campus population in order to determine the priority areas for development of solutions and technological aspects. After this stage, development divisions are determined for each large group of waste on the campus, subsequently aiming at the region's industrial reality. It has
\end{abstract}


been observed that most of the waste generated was from recyclable materials, followed by non-recyclable materials, as observed in other universities, the biggest divergence has been in the discrepancy of collected compostable material.

Keywords: Environmental; Technology; University; Compostable.

\section{Resumen}

Con el objetivo de desarrollar nuevas tecnologías que afecten directamente la realidad ambiental de Brasil en el ámbito de la gestión de residuos sólidos y la educación socioambiental, el proyecto de Síntesis es desarrollado por el Departamento de Ingeniería Química y Ambiental y por estudiantes del curso de Ingeniería Química en la Facultad de Tecnología en la Universidad Estatal de Río de Janeiro, Brasil. Para este fin, la población del campus regional de Resende, donde se desarrolla el proyecto, se utiliza como grupo focal. En función de los tipos de residuos generados por el campus, se proponen alternativas para su tratamiento, desarrollo de tecnologías que puedan aplicarse a la realidad de la sociedad brasileña y medidas que alienten a la sociedad a ser parte del proceso de tratamiento de residuos, generando un Impacto social y responsabilidad ambiental. La etapa inicial del trabajo desarrollado por el proyecto es la encuesta de los tipos de residuos generados por la población del campus para determinar las áreas prioritarias para el desarrollo de soluciones y aspectos tecnológicos. Después de esta etapa, se determinan las divisiones de desarrollo para cada gran grupo de residuos en el campus, con el objetivo de la realidad industrial de la región. Se observó que la mayoría de los desechos generados provenían de materiales reciclables, seguidos de materiales no reciclables, como se observó en otras universidades, la mayor divergencia estaba en la discrepancia del material compostable recolectado.

Palabras clave: Ambiental; Tecnología; Universidad; Compostable.

\section{Introdução}

A geração e gestão de resíduos sólidos continua sendo um problema global urgente (Smyth, 2010). De acordo com dados da Organização das Nações Unidas (ONU), no mundo, são produzidos anualmente 2 bilhões de toneladas de resíduos, sendo consequência direta do descarte inadequado e na ineficiência de uma gestão de resíduos sólidos. Dados alarmantes indicam que $99 \%$ dos produtos adquiridos pela população, são descartados em até 6 meses (Kalmykova, 2018). 
Quando estamos falando de gestão de resíduos, a colaboração da sociedade é algo essencial. Até recentemente, a gerência de resíduos era encarada como uma questão de estoque apropriado, coleta, transferência, tratamento e descarte, e o principal esforço era para minimizar os impactos ambientais e na saúde pública. Desta forma, ferramentas de engenharia e logística eram suficientes para planejar e implementar sistemas de gestão de resíduos sólidos. Mas hoje, com a introdução dos conceitos de economia circular (Mayropoulos, 2015), a administração de recursos e o comportamento social estão se tornando parte integral de qualquer sistema de gerenciamento de resíduos sólidos. Eles são essenciais para o tratamento de taxas crescentes de reciclagem e melhor qualificação dos recicláveis, para a participação de stakeholders industriais, iniciativas eco-design e ciclos fechados de produtos e materiais (Mallawarachchi, 2012; Schneider, 2018).

O Brasil, como uma economia em desenvolvimento, não está imune a esse problema. Em 2011, a população brasileira gerou 61,9 milhões de toneladas de resíduos sólidos. O crescimento dos resíduos sólidos não é o único problema, a disposição desses resíduos também é uma preocupação. 42\% do total de resíduos sólidos coletados em 2011 foram descartados de forma inadequada. Parte desta eliminação de resíduos ocorre nas regiões mais ecologicamente sensíveis do mundo (Jabbour et al. 2014). Durante a década de 1990, reciclagem e outros métodos de gestão de resíduos eram considerados os modelos mais desenvolvidos para os planejamentos de gestão municipal de resíduos sólidos (GMRS). Os modelos atuais refletem uma mudança na política onde o planejamento de resíduos saiu de uma total confiança nos aterros sanitários, e foi colocado em técnicas mais abrangentes de gestão baseadas nos princípios da gestão integrada de resíduos sólidos (GIRS) (Gabola, 1999; Kowalewski, 1999; Berger, 1999; Clift, 2000; Morrissey, 2004).

Os resíduos municipais ou urbanos são frequentemente gerados a partir de várias fontes onde as diversas atividades humanas são encontradas. Estudos indicam que a maioria dos resíduos sólidos urbanos de países em desenvolvimento são de fontes domésticas (55$80 \%$ ), seguidos por áreas comerciais (10-30\%) com quantidades variáveis de resíduos públicos, industriais, e de demais instituições (Nabegu, 2010; Nagabooshnam, 2011; OkotOkumu, 2012). Os resíduos dessas fontes são de natureza altamente heterogênea (Valkenburg et al. 2008) e possuem características físicas variáveis dependendo de suas fontes; observa-se que são compostos de resíduos alimentares, resíduos de quintal, madeira, plásticos, papéis, metais, couro, borrachas, materiais inertes, baterias, recipientes de tinta, têxteis, resíduos de construção e demolição e muitos outros difíceis de serem classificados. 
Programas abrangentes de gestão de resíduos sólidos são um dos maiores desafios para alcançar a sustentabilidade de um campus. A realização de um estudo de caracterização de resíduos é um primeiro passo crítico no planejamento bem-sucedido do gerenciamento de resíduos e no avanço da sustentabilidade geral de uma instituição de ensino superior (Smyth, 2010). Este trabalho é realizado em diversas universidades pelo mundo como na Universidade Autônoma da Baixa California (UABC) campus Mexicali I no México (Vega, 2008), Universidade de Southampton na Inglaterra (Zhang et al. 2011) e Campus Prince George da Universidade do Norte da Colúmbia Britânica (UNBC) no Canadá. Esta utilizou categorias de caracterização de resíduos adaptadas de várias metodologias de caracterização, principalmente do Estudo de Caracterização de Resíduos do Distrito Regional de Fraser George (RDFFG) e do sistema de classificação de materiais do Ministério do Meio Ambiente de Ontário de acordo com o Regulamento 102/94 (Ontario Ministry of Environment, 1994; Regional District of Fraser Fort George, 2007) superior (Smyth, 2010).

Utilizando o campus da Faculdade de Tecnologia do Estado do Rio de Janeiro (FATUERJ) como alvo, o Projeto Síntese inicialmente propõe o levantamento de dados sobre os resíduos gerados. O objetivo deste artigo é mostrar como a análise dos tipos de resíduos sólidos gerados por uma população descreve fatores importantes no sistema de gestão de resíduos sólidos e norteia as medidas a serem tomadas no processo de planejamento e estruturação do sistema de gestão.

\section{Metodologia}

Foi implementado um sistema de coleta seletiva de lixo, na faculdade de tecnologia da UERJ, a partir de um projeto de extensão chamado projeto Síntese. Para este projeto, foram produzidos cartazes, espalhados por todo o campus, para divulgação e conscientização da comunidade acadêmica sobre a importância da reciclagem e como fazer a separação dos resíduos. Sendo assim, foi feita a capacitação técnica da equipe de limpeza, e foram produzidas lixeiras a partir de containers reciclados do setor industrial, usando os conceitos de Economia Circular. Os resíduos gerados foram segregados em uma Central de Resíduos criada nesse projeto.

Para a produção das lixeiras, os containers foram compostos por bombonas fechadas de $200 \mathrm{~L}, 90 \mathrm{~cm}$ de altura e diâmetro de $57 \mathrm{~cm}$. Com uma serra Tico-Tico DW300, foi realizado um corte transversal na parte superior a fim de retirar uma tampa para a lixeira. Outro corte transversal foi realizado para diminuir a altura da bombona para $60 \mathrm{~cm}$, a fim de 
facilitar a ergonomia da lixeira. As extremidades do corte foram lixadas utilizando lixas d'água de granulometria 80. Na tampa, dois semicírculos em lados opostos foram cortados para que o resíduo pudesse ser descartado e duas abraçadeiras de nylon foram fixadas para servirem de alças, a fim de facilitar a retirada da tampa durante a coleta do resíduo. Na parte interior superior da bombona, foram colocados quatro ganchos, distribuídos uniformemente, protegidos com borracha para dar suporte à tampa e evitar que os sacos sejam rasgados, Figura 1.

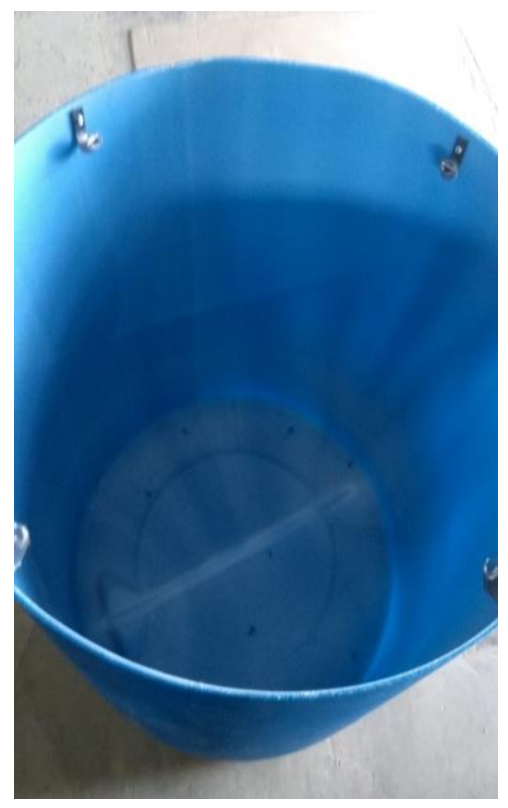

Figura 1 - Ganchos, protegidos com borracha para dar suporte à tampa

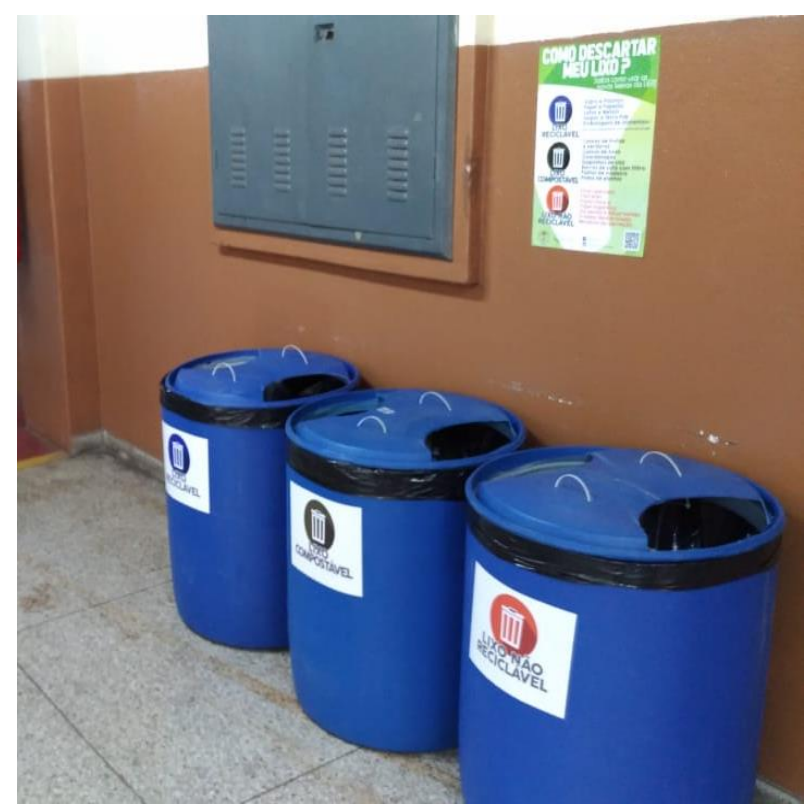

Figura 2 - Lixeiras adesivadas e colocadas em trios 
As lixeiras produzidas foram adesivadas e colocadas em trios (Figura 2), sendo um em cada corredor de maior movimento de cada andar do prédio principal do campus, que possui três andares. Um trio também foi colocada no refeitório do campus. Nos corredores, existiam lixeiras para descarte geral fixadas na parede, que após a implementação do projeto, foram convertidas em lixeiras para descarte de resíduos compostáveis e, junto a cada uma delas, uma lixeira de não-reciclável foi colocada. Esta que foi produzida através de caixas de papelão recicladas, embrulhadas com papel pardo, devidamente adesivadas. Todas as salas possuíam lixeiras de uso comum que, com a implementação do projeto, passaram a ser lixeiras para descarte de resíduo reciclável.

Os resíduos sólidos, previamente separados em sacolas, foram pesados, diariamente, durante 21 dias úteis, em uma balança mecânica modelo MIC-1C, cuja capacidade é de 150 Kg e precisão de 100 g. Após a pesagem, os resíduos não-recicláveis e compostáveis foram descartados e os resíduos recicláveis foram separados e enviados para a Associação de Catadores Recicla Resende. Os dados recolhidos foram tratados utilizando o programa Excel.

\section{Resultados e discussões}

Num período total de 45 dias, foram coletados dados efetivos de pesagem de 21 dias, enquanto 24 dias foram relativos a finais de semana, feriados e datas em que não houve funcionamento acadêmico no campus. Os dados de pesagem desses 21 dias foram plotados em gráficos explicativos, utilizando o software Microsoft Excel. Estas análises ajudam a traçar um panorama quantitativo e qualitativo dos resíduos sólidos gerados no campus da universidade.

Com uma circulação ativa de 800 pessoas no Campus da FAT, a média de resíduos sólidos gerados por dia gira em torno de $24,45 \mathrm{Kg}$, uma quantidade já esperada se tratando de um ambiente de alta circulação.

Por se tratar de um campus universitário, é esperado que os resíduos gerados refletem o cotidiano de um ambiente estudantil, onde são descartados em maiores quantidades papéis, canetas, copos descartáveis, embalagens de comidas e restos de alimentos proveniente de refeições produzidas no campus.

Os picos mais altos e mais baixos na Figura 3 podem ser considerados um indicativo da circulação de pessoas em determinado dia. Altos picos indicam uma maior geração de resíduos e, consequentemente, uma maior circulação de pessoas no campus, como, em dias de avaliações acadêmicas ou recebimento de cargas de produtos para a universidade, enquanto 
Research, Society and Development, v. 9, n. 4, e04942787, 2020

(CC BY 4.0) | ISSN 2525-3409 | DOI: http://dx.doi.org/10.33448/rsd-v9i4.2787

picos mais baixos indicam uma menor circulação de pessoas, geralmente datas próximas a feriados ou finais de semana.

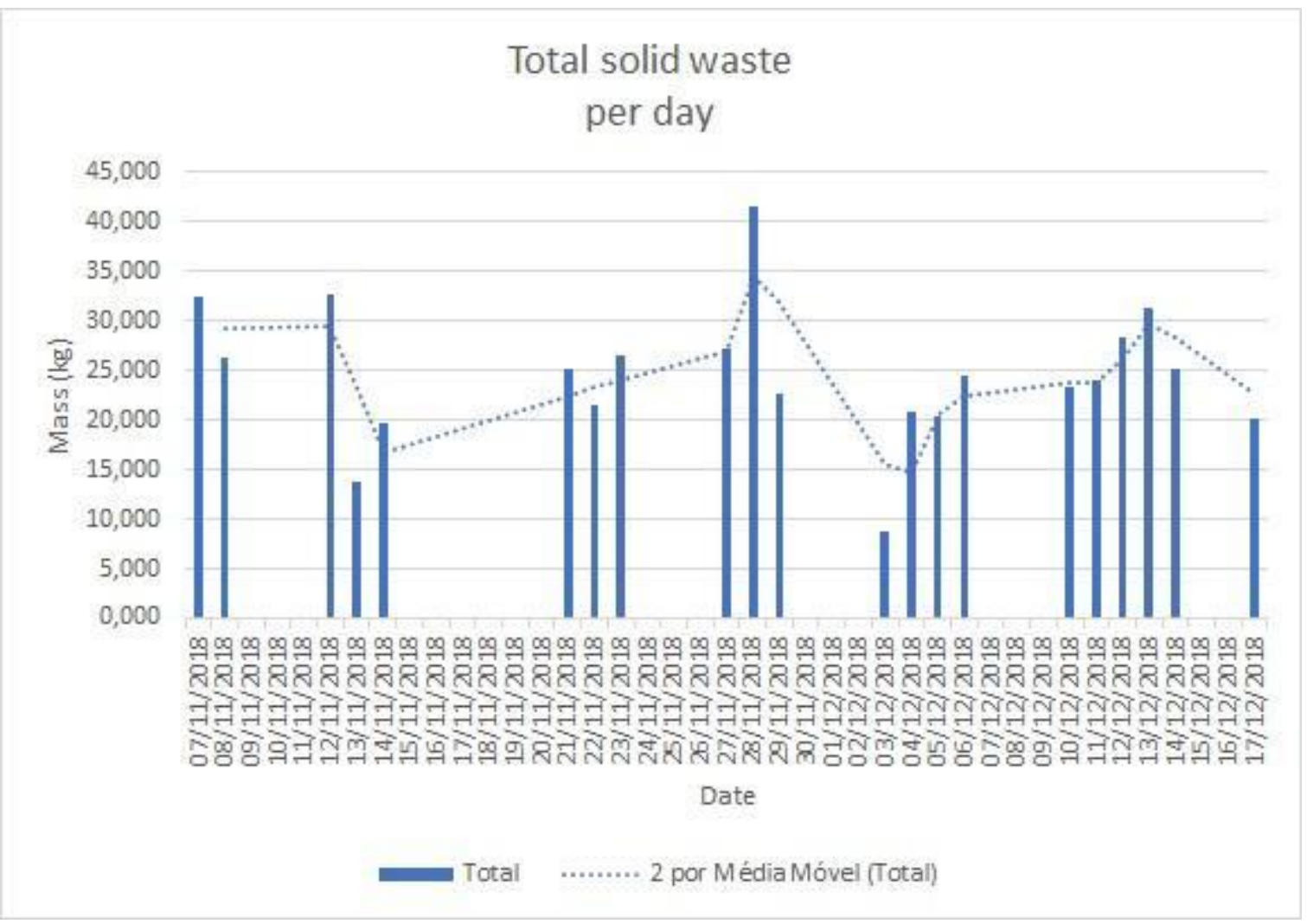

Figura 3 - Total de resíduos sólidos por dia.

Ao realizar uma análise mais detalhada dos resíduos gerados (Figura 4), tem-se o indicativo de que a maior parte do que é produzido por dia é composto por lixo reciclável e não reciclável. Sendo o primeiro composto principalmente por plásticos, papéis e papelão e o segundo majoritariamente composto por resíduos sanitários e laboratoriais.

É importante ressaltar que durante a coleta de dados, observou-se ainda uma alta proporção de mistura entre o lixo reciclável e não reciclável. Tal fato serve como medidor da responsabilidade socioambiental da população do campus e demonstra que ainda é necessária uma maior divulgação da importância da coleta seletiva e do impacto positivo que o tratamento e descarte correto desses resíduos geram na sociedade.

Tratando-se do lixo compostável, a baixa quantidade apresentada nos dados recolhidos contradiz o esperado para um campus onde um grande número de refeições é servido por dia. Mais uma vez, demonstrando uma coleta seletiva ainda ineficaz. 


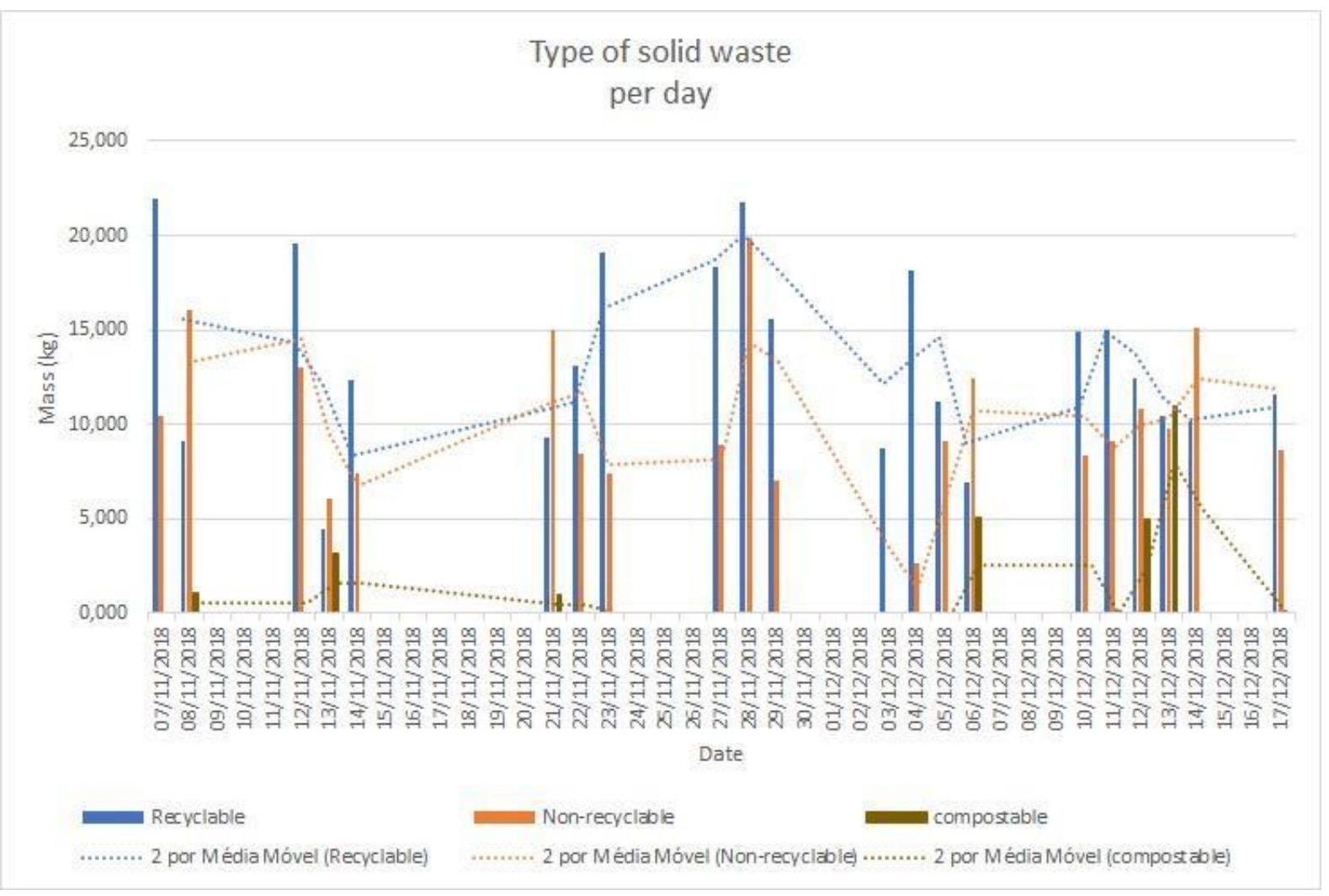

Figura 4 - Tipos de resíduos sólidos gerados por dia.

Ao final dos 45 dias da etapa de pesagem, foi possível identificar quanto de cada tipo de resíduo foi produzido durante esse período de coleta de dados. Desta forma, consegue-se mostrar que mais da metade de todo o resíduo do campus universitário em questão é, de fato, composto por lixo reciclável, seguido do lixo não reciclável e em quantidade muito menor o lixo compostável.

Uma vez que o lixo recolhido ainda é misturado, os dados representados na Figura 3 não são totalmente assertivos, no entanto, são uma base inicial necessária para elucidar um panorama ainda desconhecido no campus. É importante ressaltar que a implementação da coleta seletiva demanda uma adequação de toda a sociedade acadêmica e requer tempo para que seja completamente satisfatória e com o retorno ideal. 


\section{Types of total solid waste}

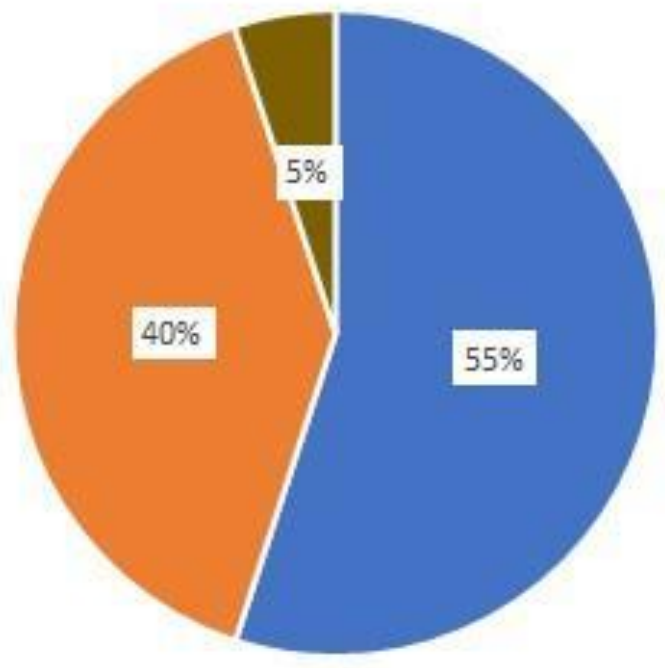

- Recyclable

- Non-recyclable

- compostable

Figura 5 - Total de resíduos sólidos gerados.

Comparando as porcentagens dos resíduos gerados no campus da FAT - UERJ (Figura 5) com os da Universidade do Norte da Colúmbia Britânica campus Prince George ${ }^{[1]}$ no qual $49,34 \%$ é reciclável, $28,42 \%$ não reciclável e $21,61 \%$ é compostável, observa-se uma similaridade no valor encontrado para o resíduo reciclável. Há, porém, uma divergência nos valores para não recicláveis e compostáveis que se dá, possivelmente, devido à diferença nos processos de recolhimento e separação.

Tendo como base os dados elucidados nos três gráficos apresentados, diversas estratégias podem ser traçadas no desenvolvimento de alternativas para o tratamento e descarte adequados dos resíduos gerados no campus. Dentre eles, podemos citar o melhor descarte do lixo reciclável. Antes da implementação da gestão de resíduos sólidos, tais resíduos eram descartados sem qualquer tipo de separação, no entanto, atualmente, a associação de catadores de Resende é contactada para dar o destino adequado aos materiais que podem ser reaproveitados. Já os resíduos que não se pode reciclar, a coleta é feita da maneira tradicional.

Além disso, uma variedade de projetos, como o desenvolvimento de composteiras, instalação de pontos de recolhimento de óleo de soja utilizado, produção de biodiesel, produção de móveis fabricados com plástico PET, dentre outros, estão sendo estudados para que sua implementação no campus seja a mais rápida possível, contando com a colaboração de alunos, professores e toda a comunidade do campus. 


\section{Conclusões}

Com o intuito de traçar um panorama da geração de resíduos sólidos do campus, os dados aqui recolhidos e analisados demonstram que uma educação socioambiental mais eficaz dos frequentadores do campus ainda é necessária para que a gestão dos resíduos seja efetiva e contínua. Ainda assim, os dados já indicam que diversos projetos voltados para a própria universidade e comunidade ao redor podem ser desenvolvidos implementando o desenvolvimento e uso de novas tecnologias voltadas para a gestão e tratamento de resíduos sólidos.

A heterogeneidade dos resíduos gerados é um grande revés em sua utilização como matéria-prima. Existe, portanto, a necessidade de fracionamento dos resíduos antes que eles possam ser submetidos a qualquer processo de tratamento significativo superior (Miezah et al. 2017).

O projeto de extensão agora se propõe a pautar, com base nos dados recolhidos, os subprojetos a serem desenvolvidos nessa área, levando em consideração quais tipos de resíduos são gerados em maior quantidade e que possam ser tratados de maneira viável no campus. Além disso, novas coletas de dados serão efetuadas para que seja possível analisar o avanço da educação e desenvolvimento tecnológico socioambiental trazido ao campus.

\section{Agradecimentos}

Os autores agradecem ao departamento de química do campus de Resende da FAT/UERJ pelo apoio e colaboração nesta pesquisa.

\section{Referências}

Berger C., Savard G., Wizere A. (1999), "EUGENE: an optimisation model for integrated regional solid waste management planning", Int. J. Environment and Pollution, 12 (2/3), 280 307.

Clift R., Doig A., Finnveden G. (2000), “The application of life cycle assessment to integrated solid waste management", Trans IChemE, 78 (B), 279-287. 
Gabola S. (1999), "Towards a sustainable basis for the EU Packaging and Packaging Waste Directive". In: ERRA Symposium November, Brussels.

Jabbour A. B. L. S., Jabbour C. J. C., Sarkis J., Govindan K. (2014), “Brazil's new national policy on solid waste: challenges and opportunities" Clean technologies and environmental policy. January 2014, volume 16, issue 1, pp 7-9.

Kalmykova Y., Sadagopan M., Rosado L. (2018),“Circular economy - from review of theories and practices to development of implementation tools", Resources, conservation and recycling, Volume 135, Pages 190-201

Kowalewski R., Lea W. R., Tittebaum M. (1999), "A standardised data reporting methodology for long-term integrated municipal solid waste management - a case study", Journal of solid waste technology and management, v. 26, (1), 26-35.

Mallawarachchi H., Karunasena G. (2012), "Electronic and electrical waste management in Sri Lanka: suggestions for national policy enhancements”, Resour Conserv Recycl, v. 68 4453.

Mayropoulos A., Tsakona M., Anthouli A. (2015), "Urban waste management and the mobile challenge", Waste management \& research, USA, vol. 33, p. 381-7.

Miezah K., Obiri-Danso K., Kádár Z., Heiske S., Fei-Baffoe B., Mensah M., Meyer A. S. (2017), "Municipal Solid Waste Management in a Low Income Economy Through Biogas and Bioethanol Production”, Waste and Biomass Valorization, volume 8, pages115-127.

Morrissey A. J., Browne J. (2004), "Waste management models and their application to sustainable waste management”, Waste Management Volume 24, Issue 3, Pages 297-308

Nabegu A. B. (2010), “An analysis of municipal solid waste in Kano metropolis, Nigeria”, J. Hum. Ecolo. 31 (2), 111-119. 
Nagabooshnam J. K. (2011), "Solid waste generation and composition in Gaborone, Botswana, Potential for resource recovery", Master thesis, Energy and environmental engineering, Department of Management Engineering, Linkoping University, Sweden.

Okot-Okumu J. (2012), "Solid waste management in African cities - East Africa", Waste Management - An Integrated Vision, ISBN: 978-953-51-0795-8, InTech, http:// dx.doi.org/10.5772/50241.

Schneider E. (2018), "Humanidade Produz Mais de 2 Bilhões de Toneladas de Lixo por Ano", ONU - Habitat. Disponível em: <https://Nacoesunidas.org/Humanidade-Produz-MaisDe-2-Bilhoes-De-Toneladas-De-Lixo-Por-Ano-Diz-Onu-Em-Dia-Mundial/> Acesso em: 20 de Janeiro de 2018.

Smyth D. P., Fredeen, A. L., Booth, A. L. (2010), "Reducing solid waste in higher education: The first step towards 'greening' a university campus", Resources, Conservation And Recycling, [s.1.], v. 54, n. 11, p.1007-1016.

Valkenburg C., Walton C. W., Thompson B. L., Gerber M. A., Jones S., Stevens D. J. (2008), "Municipal solid waste (MSW) to liquid fuels synthesis", Volume 1, Availability of feedstock and Technol. PNNL 18144, Pacific Northwest National Laboratory, Richland, WA.

Vega C. A., Benítez, S. O., Barreto M. E. R. (2008), "Solid waste characterization and recycling potential for a university campus", Waste Management [s.1.], v. 28, n. 1, p.21-26.

Zhang N., Williams I. D., Kemp S., Smith N. F. (2011), "Greening academia: Developing sustainable waste management at Higher Education Institutions", Waste Management [s.1.], v. 31, n. 7, p.1606-1616.

\section{Porcentagem de contribuição de cada autor no manuscrito}

Caroline Coutinho - $16,67 \%$

Allan Duarte - $16,67 \%$

Emanuel Carneiro - 16,67\%

Nilo A. S. Sampaio - $16,67 \%$

José Wilson de Jesus Silva - 16,67\% 
(CC BY 4.0) | ISSN 2525-3409 | DOI: http://dx.doi.org/10.33448/rsd-v9i4.2787

Carin Von Mühlen - 16,67\% 